\title{
EXAMINING THE EFFECTIVENESS OF GAMIFICATION AND GAME-BASED LEARNING IN THE CONTEXT OF BLOCKCHAIN
}

\author{
Nicholas Abela and Owen Sacco \\ Malta College of Arts, Science and Technology, Malta
}

\begin{abstract}
In the world of gaming, there has been a shift from games being used solely for entertainment, to games being used as a medium to educate. For this matter, there are two methods of making a game which are gamification or game-based learning. The former is the use of game elements, such as health points or leader boards, and they are applied to a non-gaming platform. The latter, game-based learning, includes developing a full-fledged game where the means towards the end, that is to say victory are set in a world where the player has to apply the teachings given to advance. Since this is an IT-related research, the topic chosen for this study is Blockchain, an ever-expanding sector over the past decade. Different parts of Blockchain's makeup have been dissected into small, comprehensible pieces of information in the applications created, which a brand new student to the form can take in at their own pace. Should this study go beyond this research, it would be beneficial for the experiments to have two games designed and created by someone who has the artistic and technical capabilities of creating their own assets. This would leave a better impression with the test subjects and ideally receive a better data set for examination.
\end{abstract}

\section{KEYWORDS}

Blockchain, Gamification, Game-Based Learning, Education

\section{INTRODUCTION}

This research will be investigating the effectiveness of combining teaching with video games to produce a learning environment for vast and complex topics. To conduct the experiments needed, the topic of Blockchain was selected, due to its prominence in many spheres of influence in the world. In order to educate students about the vastness of Blockchain, there needs to be a system in place. To determine which method would be most beneficial for the education of this topic, there will be a game implemented using Gamification elements, and another game that will make use of a Game-Based Learning system. In other words, this research focuses on determining whether gamification or game-based learning is most effective for teaching concepts about Blockchain. The remainder of this paper is structured as follows: section 2 provides core information about gamification, game-based learning and blockchain and reviews current work in these research areas. Section 3 provides an explanation of our methodology and Section 4 presents our results from our user study. Section 5 concludes the paper by providing an overall discussion and the future steps of this work.

\section{RELATED WORK}

In order to understand how education can be improved, there needs to be an understanding on the methods of education. (Zeng, et al., 2006) discussed an analysis of classroom-teaching in order to verify its quality at a given time. They observe how the different factors present in the classroom affect the end-result, which is how much the student learned. Among the factors that (Zeng, et al., 2006) concluded was that the need for the quality of teaching and how to motivate the students to learn better should be given its due importance, which will result in better education overall. 


\subsection{Gamification}

To understand what is needed to create a gamified application, one must understand what the term Gamification means. Coined for the digital media industry, (Deterding, et al., 2011) explains how the concept was constructed without an actual clear definition of its content. It was coined by Nick Pelling in 2002, according to (Tóth \& Tóvölgyi, 2016), but became widely known in 2010. Its most common feature is that it makes use of game-elements in a context that is not usually attributed to games, which lead this feature to become its widely accepted definition. (Deterding, et al., 2011) said that it is a concept that relates to the "game" part of a system, instead of the "play" portion. These two elements are what defines a game created for leisure or a system created with game-elements added on top of it to be made more appealing to customers or students.

As stated before, in order for a system to be considered as a gamified one, it cannot be design as a full game, but rather as a system that takes certain elements into itself to make the functional aspects of the system less tedious or more educative. (Tóth \& Tóvölgyi, 2016) gives various examples of what elements can be considered for such a system. The examples below are just a few from the ones mentioned:

- Points: The most basic of reward systems, points are given when the user performs an action that reflect the application's functionality.

- Badges: A symbol of achievement, the badge is used to display the successes of the player's actions. These are comparable to the badges handed out by Boy Scouts.

- Avatar: The virtual representation of the player online, this element is used for the social aspect of such systems.

- Progress Bar: A system that records the experience gained by actions completed, and translates it into visual feedback for the player to follow.

As outlined by (Codish \& Ravid, 2014), in order for a system to properly implement gamification, the system itself must show a degree of adaptability, so that each player feels comfortable within the community of the service. This adaptability must be taken from different angles, between elements such as game mechanics and dynamics, or personality and social demographics.

There are multiple sectors in society that make use of such systems. Once again, (Tóth \& Tóvölgyi, 2016) offers some examples, some of which are:

- Education: This can be seen in Duolingo and Codecademy, where the former is a language teaching system, whereas the latter is used by programmers to learn their craft. These two systems make use of the aforementioned game elements that (Tóth \& Tóvölgyi, 2016) listed, which include experience points, points that are presented as currency and badges to showcase progress.

- Tasks: Gamification helps people accomplish their goals on the Habitica platform just as their slogan is: Inspire yourself to do something. The website does this rewarding active members with gold and experience, and inactive ones with loss of health. Furthermore, every user can build an avatar on the website, but some features are only available via coins and gold. Thus users can "spend" the gold they have collected.

\subsection{Game-Based Learning}

Contrary to Gamification, Game-Based Learning is the use of a serious game in order to create a learning environment. (Dunwell, et al., 2011) discusses the use of serious game and explains what needs to blend the game with education. It is very important to note that a serious game cannot fully be a permanent replacement of traditional methods of education. The crucial aspect of such a type of game is that it can add to the learning environment of students, and aid teachers in explaining better their subject.

In their report, (Dunwell, et al., 2011) create a case study which was EU-Funded in order to showcase how effective a game is at teaching its participants. The game featured a 3D simulation of an evacuation scenario, where the player needs to escape from a burning building. Alternatively, 3D games are not the only way of creating an educative game. (Hung, et al., 2014) created a multi-touch puzzle which teaches primary school students the geographical concepts of Taiwan. In his research, (Petrov, 2014) looked at using Minecraft's educational features to see how effective they truly are. In his research, he collaborated with different teachers who made use of this game in order to teach younger students. 


\subsection{Blockchain}

In 2008, Satoshi Nakamoto released the paper "Bitcoin: A Peer-To-Peer Electronic Cash System", which paved the way for the popular blockchain system. (Srivastava, et al., 2019) gave a detailed rundown on how the system works.

A blockchain is an increasing set of data blocks that are connected in the form of a single long chain. These blocks are connected by a secure cryptographic hash. This chain of linked data blocks is viewed as a general ledger and is spread over a peer to peer network. Every computer that is connected to the network has an updated copy of the same general ledger. Most importantly, the ledger itself is secure, and the database can be extended only by adding new blocks into the chain. Changing records which have already been assigned to the chain is impossible.

In order to understand how blockchain works, (Srivastava, et al., 2019) explain the 4 main points of its security.

- Data encryption: There are two types of encryption schemes for various purposes: Symmetric and Asymmetric. In asymmetric algorithms, various keys are used for plaintext encryption and ciphertext decryption. Sender and receiver in this form of encryption both have a key pair of a public and private key. Because the data is encrypted by public key recipients, it is only the recipient who can decrypt the data.

- Modification prevention: The data stored in the chain is not easy to change. Using a Hash Reference, the blocks are locked together and each new block contains previous block's hash value. A hash function's main property is that if anyone changes a single bit in the input, the entire value will change in output.

- Inserting new blocks: Bitcoin mining is the process of adding transaction records to the public ledger of past transactions. The miners solve a complex mathematical problem referred to as 'Proof of Work' to add a new block. In the same network, there may be many miners, but only one can be successful at any given time.

- Data verification in blocks: Inside a block the data or transactions are stored in the form of a hash-based tree data structure called a Merkle Tree. If someone does not follow the structure of the tree, and a single transaction is already manipulated, then the entire hash output will be different. Only those transactions that have been tampered with will be incorrect, whereas the rest will be the same.

The most famous use of blockchain is the cryptocurrency Bitcoin which was released in 2009. Bitcoin operates under blockchain technology and follows the same regulations and rules. In order to store money a user needs an online wallet, which contains a collection of software-controlled Bitcoin addresses and private keys. A private key is a cryptographic hash of 64 characters which proves ownership of a specific address.

(Latifi, et al., 2019) also provide a practical use for blockchain, this time within the sphere of Real Estate. The currency used by this research is not Bitcoin, but Ethereum, which makes use of Ether. Ethereum is another cryptocurrency that stemmed from Bitcoin. The value of this cryptocurrency is far less than its predecessor.

\section{METHODOLOGY}

In the proposed experiment, the implementations created will be used to lead the study towards an understanding on how effective the games are in actuality. The first step is to create a design which will benefit the respective game's genre. Each game has its own unique function, one assigned to Game-Based Learning and another to Gamification. The implemented Gamification-based learning game is accessible here $^{1}$ and the Game-based learning game is accessible here ${ }^{2}$.

\footnotetext{
${ }^{1}$ Gamification-based learning game: https://bit.ly/2YJNhWG

${ }^{2}$ Game-based learning game: https://bit.ly/37CTsQc
} 


\subsection{Technologies Used}

As this is a game related experiment, the choice of game-engine is paramount. The final choice of engine needs to be taken with respect to the experience the developer has with it, the effectiveness of the game-engine in the given two scenarios and the ease of which a game can be built and published to be distributed among the test subjects. After careful consideration of these facts, Unity was the choice of engine.

The games were created on a WebGL Unity build option which, by the end of the development life cycle, allows for a deployment on an online website. The WebGL build option creates a folder with an index.html link, compiled build data and Unity's TemplateData, which can be seen at the start of the game. This choice was taken because of the ease of distribution a game hosted on a website offers.

\subsection{Gamification Design}

(Anam Fathoni \& Delima, 2016) explained that game elements can be re-purposed to achieve the goal of educating a person in a given subject. In this implementation, the elements that were added were progression through multiple stages, points awarded for correct questions, collection of resources, randomly generated avatars and an end screen showing what was achieved and what was not. Added to these elements was a narrative that gave movement to the game. As (Palomino, et al., 2019) discussed, the use of narrative can be implemented in order to help the user build their own experience, within the logic of the game. To create the game, an asset called Fungus was used to create objects such as menus and another asset called "2000+ Faces" was used to create a randomly generated avatar for the player. The player is then introduced to a brief history of what is currently trending around the world. The narrative has a negative undertone, suggesting that the avatar's system is being hacked. This gives the player the sense of being part of the situation by being the character themselves. It also gives the game a more interactive feel, rather than having to rely on simple instructions. This is followed by an explanation of the game's reward system. The game's winning and losing conditions are tied to the player's health points, and the keys received.

The following rules are applied to the game:

- A player has up to three chances to guess a question wrong throughout the game.

- A player can progress to the next level if at least two keys are attained.

- If at any given level, the player misses two keys, even if they still have 3 chances, the game is over. This gives the player a sense of urgency to make sure that the game is played attentively.

The player is given three sections of information, each section given at different stages of the game. They are then given the choice to either attempt the questions, or re-read the information. Each question was given two variables, later used as part of the data set, which were the "Attempted" and "Correct" variables. If the three questions were attempted but only one was correct, it can be safely assumed that the player lost the game. If two of the questions were correct, the player could proceed without having to answer the third question. The result sheet also highlights what keys the player managed to attain, so that when the game is played again, extra attention is given to the sections that were not fully understood. The game will go through the loop of supplying information, waiting for answers and progressing three times.

\subsection{Game-Based Learning Design}

Compared to the Gamification section, this game was made possible using a complex game engine which can be imported into Unity, called TopDown Engine, where the character within the game is an orange rectangular blob. Instead of having a start button, the game launches playing already, with two simple instructions given to the player. These are "Press WASD to move" and "Press SPACE to interact with doors." In this game, the player is transported to another room, where an instruction sheet is given. The questions are are allocated to a set of coloured doors, which are used as the modes of transport between rooms. Green and Blue doors offer progress forward or backwards, whereas Red doors offer either victory or defeat. This setup was made to assess how well the player does when faced with familiar information, given that proper implementation was given previously. The player can move on the instructions and read at their own pace, and a timer starts counting up, which will be used in the final analysis of the data. 
This game has a different set of rules:

- A player can go back and forth through questions and instruction sheets to read at their own pace.

- If a player answers incorrectly, they are transported to a room with a red door, which will end the game in defeat.

- The game is won if the player makes it through all 9 questions and goes through the red door at the end, which means victory this time round.

\subsection{Data Collection}

In order to collect data, the subjects chosen all varied in age, education and IT experience as much as possible. The chosen subjects will be ranging from 18 to 35 years of age, with different IT experience, as well as different takes on gaming itself. The design of the game was done with the intention that someone with no prior gaming or IT expertise could pick up and play till the end. Gender was not a criteria that would be mentioned in any questionnaire, due to gender not being a factor considered in the results. For both games, if the method of distribution was chosen correctly, then the Gamification portion should prove a high attempted is to correct ratio. The Game-based learning portion should provide with low timer numbers.

After trying both games, a questionnaire was supplied to the test subjects, targeting both quantitative and qualitative data. The former was targeted using a 10-point rating scale, where 1 indicated the least favourable outcome and 10 for the best outcome. The latter was gathered using two opinion based questions.

\section{RESULTS AND DISCUSSION}

In this section, the results for both games are defined and examined.

\subsection{Gamification}

Table 1 shows the frequency of the attempts and correct answers throughout the whole experiment. Overall, the results show a positive number of attempts, and a high number of correct answers as well. Based on this data, since the game required player to answer two questions correctly to progress, it can be deducted that only one player failed the game, and that this player failed during the first section.

According to the data, only $441 \%$ of the questions had an attempt rate equal to the correctly answered rate, whereas $331 \%$ of the questions had a two more attempts than correct answers, and $231 \%$ had only a one attempt difference to correct answers.

Table 1. Frequency of attempts and correct answers

\begin{tabular}{ccc}
\hline Question ID & Attempted & Correct \\
\hline S1_Q1 & 9 & 7 \\
S1_Q2 & 9 & 8 \\
S1_Q3 & 10 & 8 \\
\hline S2_Q1 & 9 & 9 \\
S2_Q2 & 8 & 8 \\
S2_Q3 & 9 & 8 \\
\hline S3_Q1 & 9 & 9 \\
S3_Q2 & 9 & 7 \\
S3_Q3 & 8 & 8 \\
\hline
\end{tabular}




\subsection{Game-Based Learning}

Table 2 below shows the frequency of the type of message which was shown, as well as the amount of time taken to be displayed said message. In the table, the victory screen will be an represented by the letter "V", and the losing screen will be represented by the letter "L". Through the data gathered, it can be deduced that something was amiss in the experiment. Having only $10 \backslash \%$ of the dataset being victorious, it can be concluded that the game did not successful deliver all the information it was capable of. Each player took an average of 195.4 seconds, around 3-4 minutes, to play the game.

Table 2. Player's duration in the game and the result

\begin{tabular}{ccc}
\hline Player ID & Time (Seconds) & Message Shown \\
\hline Player 1 & 170 & $\mathrm{~V}$ \\
Player 2 & 76 & $\mathrm{~L}$ \\
Player 3 & 237 & $\mathrm{~L}$ \\
Player 4 & 139 & $\mathrm{~L}$ \\
Player 5 & 165 & $\mathrm{~L}$ \\
Player 6 & 125 & $\mathrm{~L}$ \\
Player 7 & 370 & $\mathrm{~L}$ \\
Player 8 & 152 & $\mathrm{~L}$ \\
Player 9 & 192 & $\mathrm{~L}$ \\
Player 10 & 278 & $\mathrm{~L}$ \\
\hline
\end{tabular}

\subsection{Discussion}

The results provided by the test subjects prove that, based solely on this test between Gamification and Game-Based Learning, it is Gamification that ought to be implemented when tackling a new, complex subject. This deduction is made in light of the two systems being implemented as they were in the experiment. Had further design and development choices been made, such as the ones provided by the opinions of the test subjects, the results would have surely been very different.

\section{CONCLUSION}

In this study, two implementations were applied and tested to determine what is more effective, Gamification or Game-based learning. The games where handed out to random people from different backgrounds, in order to determine if the developed systems could provide a good enough basis for the topic chosen, which was Blockchain.

Based on the results, Gamification managed to elicit an overall better result from the test subject than Game-Based Learning. In the experiments provided, gamified system managed to have better overall scoring than its counterpart. The Gamification system managed to have a 90\\%-win rate from the test subjects, while the direct opposite was observed in Game-Based Learning, with only a 101\%-win rate achieved. The reason for this discrepancy in victories for the second system can be found within the opinions of the test subjects, who gave substantial feedback in ways to improve the system.

The Game-Based learning implementation received praise on its aesthetic qualities. It did not fulfil the functional requirement of teaching someone or provide enjoyment for the player. More RPG (Role Playing Games) elements, such as questing, monster killing and NPC transactions could be made to distribute the information, which makes it more interesting.

Gamification was developed to have multiple text areas the student must click through. Future implementations need to address the reading issue of these types of games. In reality, reading is an essential part of learning, but the way the players are made to read can make or break the game. 


\section{REFERENCES}

Anam Fathoni, A. F. C. \& Delima, D., 2016. Gamification of learning kanji with "Musou Roman" game. $20161 s t$ International Conference on Game, Game Art, and Gamification (ICGGAG), pp. 1-3.

Codish, D. \& Ravid, G., 2014. Adaptive approach for gamification optimization. IEEE/ACM 7th International Conference on Utility and Cloud Computing, pp. 609-610.

Deterding, S., Dixon, D., Khaled, R. \& Nacke, L., 2011. From game design elements to gamefulness: defining" gamification. Proceedings of the 15th international academic MindTrek conference: Envisioning future media environments, pp. 9-15.

Dunwell, I. et al., 2011. Blended Game-Based Learning Environments: Extending a Serious Game into a Learning Content Management System. 2011 Third International Conference on Intelligent Networking and Collaborative Systems, pp. 830-835.

Hung, C.-Y., Kuo, F.-O., Sun , J. C.-Y. \& Yu, P.-T., 2014. An Interactive Game Approach for Improving Students' Learning Performance in Multi-Touch Game-Based Learning. IEEE Transactions on Learning Technologies, pp. 31-37.

Latifi, S., Zhang, Y. \& Cheng, L.-C., 2019. Blockchain-Based Real Estate Market: One Method for Applying Blockchain Technology in Commercial Real Estate Market. 2019 IEEE International Conference on Blockchain (Blockchain), pp. 528-535.

Palomino, P. T. et al., 2019. Narrative for Gamification in Education: Why Should you Care? 2019 IEEE 19th International Conference on Advanced Learning Technologies (ICALT), pp. 97-99.

Petrov, A., 2014. Using Minecraft in Education: A Qualitative Study on Benefits and Challenges of Game-Based Education. Master Thesis, University of Toronto, Toronto, Canada.

Srivastava, G., Dhar, S., Dwivedi, A. D. \& Crichigno, J., 2019. Blockchain Education. 2019 IEEE Canadian Conference of Electrical and Computer Engineering (CCECE), pp. 1-5.

Tóth, Á. \& Tóvölgyi, S., 2016. The introduction of gamification: A review paper about the applied gamification in the smartphone applications.. 7th IEEE International Conference on Cognitive Infocommunications (CogInfoCom), pp. 213-218.

Zeng, Z., Huang, C. \& Yue, D., 2006. Research on Classroom-Teaching Quality and Its Evaluation index System of Higher Education. IEEE International Conference on Service Operations and Logistics, and Informatics, pp. 118-122. 